\title{
The linear sampling method for three-dimensional inverse scattering problems
}

David Colton* Klaus Giebermann ${ }^{\dagger} \quad$ Peter Monk* $^{*}$

(Received 7 August 2000)

\begin{abstract}
The inverse scattering problem under consideration is to determine the shape of an obstacle in $\mathrm{R}^{3}$ from a knowledge of the time harmonic incident acoustic wave and the far field pattern of the scattered wave with frequency in the resonance region. A method for solving this
\end{abstract}

*Department of Mathematical Sciences, University of Delaware, Newark, Delaware 19716, USA.

†Institut für Angewandte Mathematik, Universität Bonn, 53115 Bonn, GERMANy, mailto:gieberma@iam.uni-bonn.de.

${ }^{0}$ See http: //anziamj . austms.org. au/V42/CTAC99/Colt for this article and ancillary services, (c) Austral. Mathematical Soc. 2000. Published 27 Nov 2000. 
nonlinear and improperly posed problem is presented which is based on solving a linear integral equation of the first kind and avoids the use of nonlinear optimization methods. Numerical examples are given showing the practicality of this new approach.

\section{Contents}

1 Introduction

C436

2 Inverse Obstacle Scattering Problems

C437

3 The Linear Sampling Method for Solving Inverse Scattering Problems

C440

4 Numerical Realization

C443

$5 \quad$ Numerical Results

C451

6 Conclusion

$\mathrm{C} 454$

References

C457 


\section{Introduction}

Inverse scattering theory is concerned with determining the shape of a scattering obstacle from a knowledge of the scattered acoustic or electromagnetic wave corresponding to a given incident field. Although of considerable importance in various areas of science and technology, the mathematical and numerical analysis of such problems is of relatively recent origin. There have been a number of successful reconstruction algorithms proposed for the three dimensional time harmonic inverse scattering problem for both acoustic and electromagnetic waves, all of which are based on some type of nonlinear optimization scheme $[4,1,9,12,13,15]$. Such schemes are particularly attractive since they are able to treat the nonlinear and improperly posed nature of the inverse scattering problem in a simple and straightforward manner [3, 14].

Unfortunately, nonlinear optimization methods are often unsatisfactory in practical shape reconstruction problems. This is due to the fact that in order to implement such methods it is necessary to know the number of components of the scatterer as well as a rough idea of the geometry of each component in order to choose an appropriate parameterization of the surface. In addition, it is also necessary to know the boundary condition satisfied by the field on the surface of the scatterer. The fact that in many cases of practical importance some or all of the above a priori information is not available has motivated the development in recent years of methods of inversion that are not based on nonlinear optimization methods $[2,5,16,17,18]$. An attractive feature of these new methods is that they require no a priori infor- 
mation at all about the connectivity or geometry of the scattering obstacle. In addition, the method originally proposed by Colton and Kirsch [2] and improved by Colton, Piana and Potthast in [5] also requires no knowledge of the boundary conditions satisfied by the field on the boundary of the scatterer (see also [16]). A numerical implementation of the linear sampling method for three dimensional problems was presented by Colton, Giebermann and Monk [7]. The purpose of this paper is to report on further numerical improvements of the linear sampling method as well as their application to inverse scattering problems involving penetrable, inhomogeneous, isotropic media.

The plan of our paper is as follows. In the next section we will describe the inverse scattering problem to be studied. Then we present the linear sampling method for solving this problem. We will then discuss the numerical implementation of this method and finally we will present some numerical results obtained by the linear sampling method.

\section{Inverse Obstacle Scattering Problems}

The aim of this section is to provide some basic facts about the mathematical theory of wave scattering in homogeneous and inhomogeneous media (see [3]). We start with the homogeneous case: Let $\Omega \subset \mathrm{R}^{3}$ be the open complement of an unbounded smooth domain and let $\Gamma=\partial \Omega$ be the boundary of $\Omega$. Since we will only consider time-harmonic waves, we can identify an acoustic 
wave with a complex valued function that does only depend on space and not on time. The most important physical parameter for this model is the wavenumber $k$, defined as $k=2 \pi / \lambda$, where $\lambda$ denotes the wavelength.

If we denote an incoming wave by $u^{i}$, the scattered wave by $u^{s}$ and the total wave by $u=u^{i}+u^{s}$, then the scattering process leads to an exterior boundary value problem for the Helmholtz equation:

$$
\Delta u+k^{2} u=0 \quad \text { in } \quad \mathrm{R}^{3} \backslash \bar{\Omega}
$$

where the boundary values on $\Gamma$ depend on the physical properties of the scatterer $\Omega$, i.e., $u^{i}+u^{s}=0$ on $\Gamma$ for "sound soft" and $\frac{\partial}{\partial \nu}\left(u^{i}+u^{s}\right)=0$ on $\Gamma$ for "sound hard" scatterer. Furthermore, the scattered wave $u^{s}$ has to satisfy the Sommerfeld radiation condition

$$
r\left(\frac{\partial}{\partial r}-i k\right) u^{s} \rightarrow 0 \text { for } \quad r=|x| \rightarrow \infty .
$$

The radiation condition (2) yields the asymptotic

$$
u^{s}(x)=\frac{e^{i k|x|}}{|x|}\left\{u_{\infty}(\widehat{x})+\mathcal{O}\left(\frac{1}{|x|^{2}}\right)\right\} \quad \text { for } \quad|x| \rightarrow \infty
$$

where $u_{\infty}$ is the "farfield pattern" defined on the unit sphere $S^{2}=\{x \in$ $\left.\mathrm{R}^{3}:|x|=1\right\}$. Figure 1 illustrates the decomposition of an acoustic field in an incoming and a scattered field. Here, the incoming wave is a plane wave travelling in the positive $x$-direction. We now come to the inhomogeneous 
scattering of a plane wave on a sound soft obstacle

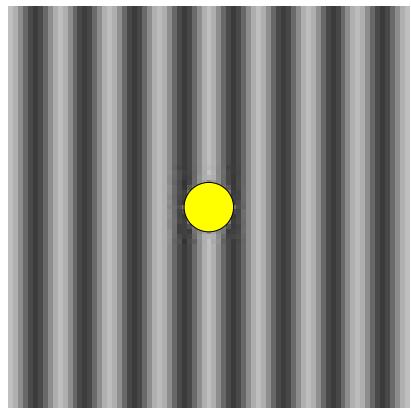

$\operatorname{Re}\left(u^{i}\right)$

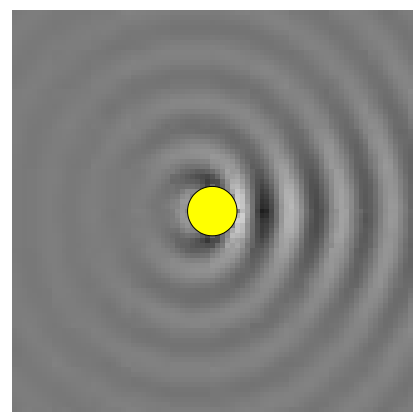

$\operatorname{Re}\left(u^{s}\right)$

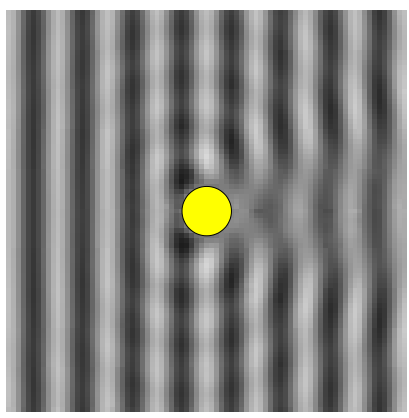

$$
\operatorname{Re}\left(u^{i}+u^{s}\right)
$$

scattering of a plane wave on a sound hard obstacle

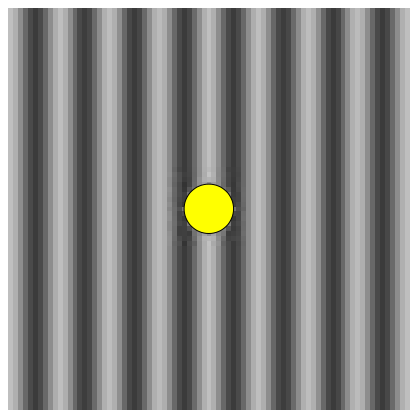

$\operatorname{Re}\left(u^{i}\right)$

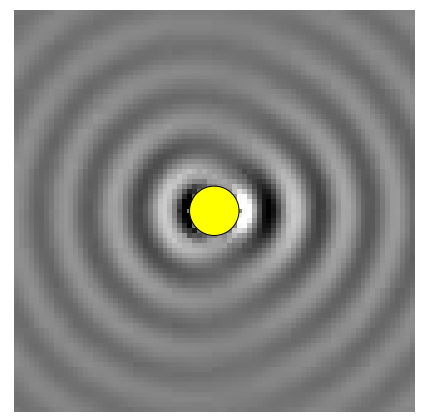

$\operatorname{Re}\left(u^{s}\right)$

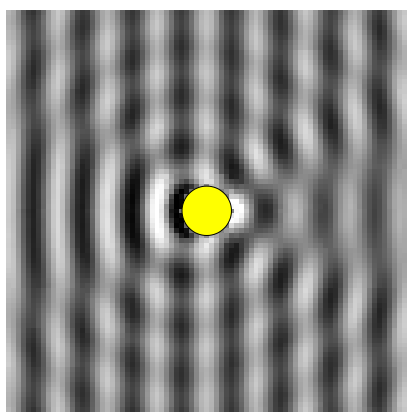

$$
\operatorname{Re}\left(u^{i}+u^{s}\right)
$$

Figure 1: Here we show the decomposition of the acoustic field in an incoming and a scattered field 
case, where we assume that we have a medium with a constant density in $\mathrm{R}^{3} \backslash \bar{\Omega}$ and thus a constant wavenumber $k$. Inside the obstacle $\Omega$ the density and hence the wavenumber may vary. The mathematical formulation of the scattering of an incoming wave $u^{i}$ reads as

$$
\left\{\begin{array}{c}
\Delta u+k^{2} n(x) u=0 \quad \text { in } \mathrm{R}^{3}, \\
u=u^{i}+u^{s} \\
\lim _{r \rightarrow \infty} r\left(\partial u^{s} / \partial r-i k u^{s}\right)=0
\end{array}\right.
$$

where $n(x)$ denotes the "refractive index". Again, the radiation condition yields the asymptotic (3).

The inverse obstacle scattering problem then reads as follows: given one or more incident plane waves $u^{i}(x ; \widehat{\theta}):=e^{i k x \cdot \widehat{\theta}}$, travelling in the directions $\widehat{\theta} \in \mathrm{S}^{2}$ and the associated farfield pattern $u_{\infty}(\widehat{x} ; \widehat{\theta}), \widehat{x} \in \mathrm{S}^{2}$ we want to determine the shape $\Omega$ of the scatterer. Note that due to Rellichs lemma, a radiating solution of the Helmholtz equation is uniquely determined by its farfield pattern.

\section{The Linear Sampling Method for Solving Inverse Scattering Problems}

In this section we briefly describe the linear sampling method for the solution of inverse obstacle scattering problems (see also $[2,5,6,7]$ ). 


\section{The Linear Sampling Method for Solving Inverse Scattering Problems C441}

Let $F: L^{2}\left(\mathrm{~S}^{2}\right) \rightarrow L^{2}\left(\mathrm{~S}^{2}\right)$ denote the farfield operator defined as

$$
(F g)(\widehat{x})=\int_{\mathrm{S}^{2}} u_{\infty}(\widehat{x} ; \widehat{\theta}) g(\widehat{\theta}) d s(\widehat{\theta}) .
$$

Note that the farfield pattern $u_{\infty}(\cdot ; \cdot)$ serves as the kernel of the integral operator $F$ and that the range of the operator is dense in $L^{2}\left(\mathrm{~S}^{2}\right)$. Furthermore, let us consider the singular value decomposition of the operator $F$, i.e., we have two complete orthonormal bases $V=\left\{v_{1}, v_{2}, \ldots\right\}$ and $U=\left\{u_{1}, u_{2}, \ldots\right\}$ of $L^{2}\left(\mathrm{~S}^{2}\right)$ and a non increasing sequence of singular values $\sigma_{1} \geq \sigma_{2} \geq \ldots$, such that

$$
F v_{j}=\sigma_{j} u_{j}, \quad j=1,2, \ldots
$$

holds. Using the orthonormal bases $U$ and $V$ we can define the finite dimensional spaces $U_{n}:=\operatorname{span}\left\{u_{1}, u_{2}, \ldots, u_{n}\right\}$ and $V_{n}:=\operatorname{span}\left\{v_{1}, v_{2}, \ldots, v_{n}\right\}$ as well as the $L^{2}\left(\mathrm{~S}^{2}\right)$ orthogonal projections $\Pi_{n}^{(U)}$ and $\Pi_{n}^{(V)}$ on these spaces:

$$
\Pi_{n}^{(U)} f=\sum_{j=1}^{n}\left\langle f, u_{j}\right\rangle u_{j} \quad \text { and } \quad \Pi_{n}^{(V)} f=\sum_{j=1}^{n}\left\langle f, v_{j}\right\rangle v_{j} .
$$

Let $\Psi_{z}$ denote a point source located at $z \in \mathrm{R}^{3}$ and $r_{z}$ its farfield pattern, i.e.,

$$
\Psi_{z}(x)=\frac{e^{i k|x-z|}}{|x-z|} \quad \text { and } \quad r_{z}(\widehat{x})=e^{-i k \widehat{x} \cdot z} .
$$




\section{The Linear Sampling Method for Solving Inverse Scattering Problems C442}

The idea of the linear sampling method is to look at the equation

$$
F g_{z}^{n}=\Pi_{n}^{(U)} r_{z}=: r_{z}^{n} .
$$

The motivation is that for $z \notin \Omega$ the resulting farfield pattern $r_{z}$ is "non physical" in the sense that we cannot continuate $\Psi_{z}$ from the boundary $\Gamma$ to a radiating solution of the Helmholtz equation in $R^{3} \backslash \bar{\Omega}$. In contrast, if $z$ lies inside $\Omega$ then we can find such a continuation which is $\Psi_{z}$ itself. Hence, we hope to observe the physical sensibility of the right hand side, which depends on $z$, from the norm of the solution. Note that since we look at finite dimensional projections, the equation (9) is always solvable. Hence, we use the norm of the solution as an indicator. More precisely, the method is based upon the following observation: for any two points $z \in \Omega$ and $\widetilde{z} \in \mathrm{R}^{3} \backslash \bar{\Omega}$ holds

$$
\lim _{n \rightarrow \infty} \frac{\left\|g_{z}^{n}\right\|}{\left\|g_{\tilde{z}}^{n}\right\|}=0
$$

For $n \in \mathrm{N}$ we define

$$
M_{n}:=\min _{z \in \mathrm{R}^{3}}\left\|g_{z}^{n}\right\|_{L^{2}}
$$

and approximate the scatterer $\Omega$ by $\Omega_{n}^{\varepsilon}, 0<\varepsilon<1$, defined as

$$
\Omega_{n}^{\varepsilon}:=\left\{z \in \mathrm{R}^{3}: \frac{M_{n}}{\left\|g_{z}^{n}\right\|_{L^{2}}} \geq \varepsilon\right\} .
$$

Since the integral equation (9) is ill posed, regularization is necessary. Several modifications of the method have been proposed (see $[5,11]$ ). For our numerical examples, we use the following two variants: 
(I) Tikhonov regularization of (9), i.e.,

$$
\left(F^{*} F+\alpha I\right) g_{z}^{n}=F^{*} r_{z}^{n} .
$$

The regularization parameter $\alpha$ is obtained by Morozovs discrepancy principle [19] where the parameter $\delta$ is an estimate for the error in the data:

$$
\left\|F^{*} F g_{z}^{n}-r_{z}^{n}\right\|=\delta\left\|g_{z}^{n}\right\|
$$

(II) Modified integral equation $[10,11]$

$$
\left(F^{*} F\right)^{1 / 4} g_{z}^{n}=\Pi_{n}^{(V)} r_{z} .
$$

\section{Numerical Realization}

For the numerical realization of the linear sampling method we assume that an approximate "measured" far field pattern $u_{\infty}^{a}$ is known for $n$ incident plane waves with direction vectors $\widehat{\theta}_{j} \in \mathrm{S}^{2}, j=1, \ldots, n$, and measured in the same directions. Thus we have as data

$$
\left\{u_{\infty}^{a}\left(\widehat{\theta}_{j}, \widehat{\theta}_{\ell}\right)\right\}_{j, \ell=1}^{n}
$$

and $u_{\infty}^{a}\left(\widehat{\theta}_{j}, \widehat{\theta}_{\ell}\right)$ is approximately equal to $u_{\infty}\left(\widehat{\theta}_{j}, \widehat{\theta}_{\ell}\right)$ (we shall make this precise shortly). In our numerical examples we take $n \in\{42,162\}$ and $\widehat{\theta}_{j}, j=$ 

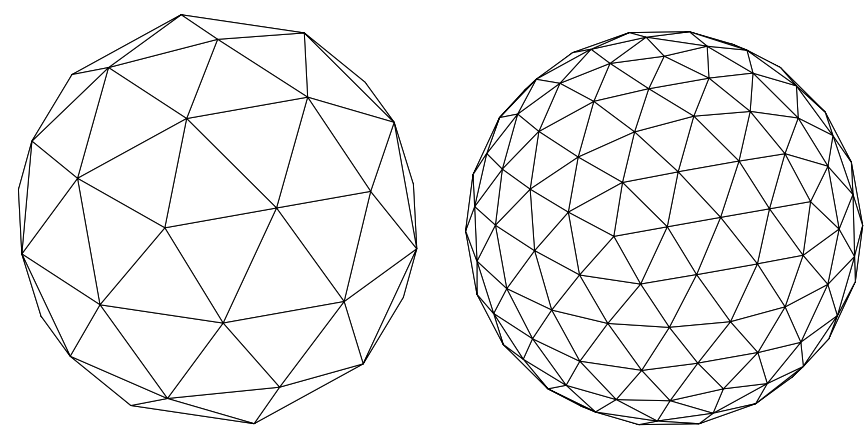

Figure 2: Here we show two surface triangulations of the unit sphere $\mathrm{S}^{2}$ on which measurements are taken (in the far field). They use 80 triangles with 42 nodes respectively 320 triangles with 162 nodes. 
$1, \ldots, n$, are located at the nodes of the surface triangulations of $\mathrm{S}^{2}$ shown in Figure 2. As the kernel of the farfield operator $F$ is smooth, we can use a Nyström type method for the numerical approximation of the integral operator, that is,

$$
(F g)\left(\widehat{\theta}_{j}\right) \approx \frac{4 \pi}{n} \sum_{\ell=1}^{n} u_{\infty}^{a}\left(\widehat{\theta}_{j}, \widehat{\theta}_{\ell}\right) g\left(\widehat{\theta}_{\ell}\right)=\mathbf{F g}
$$

with the matrix

$$
\mathbf{F}=\left(\frac{4 \pi}{n} u_{\infty}^{a}\left(\widehat{\theta}_{j}, \widehat{\theta}_{\ell}\right)\right)_{j, \ell=1}^{n}
$$

and a vector

$$
\mathbf{g}=\left(g\left(\widehat{\theta}_{1}\right), g\left(\widehat{\theta}_{2}\right), \ldots, g\left(\widehat{\theta}_{n}\right)\right)^{\top}
$$

Furthermore, we approximate the $L^{2}\left(\mathrm{~S}^{2}\right)$ norm for $g \in L^{2}\left(\mathrm{~S}^{2}\right)$ by the Euclidian norm of the vector $\mathbf{g}$, i.e., $\|g\|_{L^{2}\left(\mathrm{~S}^{2}\right)} \approx \sqrt{4 \pi / n}\|\mathbf{g}\|_{\ell^{2}}$.

The linear sampling method starts by computing the singular value decomposition of the matrix $\mathbf{F}$

$$
\mathbf{F}=U \Lambda V^{*}
$$

where $U$ and $V$ are unitary and $\Lambda$ is real diagonal with $\Lambda_{\ell, \ell}=\sigma_{\ell}, 1 \leq \ell \leq n$. 
The solution of (9) is then equivalent to solving

$$
\Lambda V^{*} \mathbf{g}_{z}=\rho_{z},
$$

with

$$
\rho_{z}=\left(\rho_{z, 1}, \rho_{z, 2}, \cdots, \rho_{z, n}\right)^{\top}=U^{*} \mathbf{r}_{z}
$$

and

$$
\mathbf{r}_{z}=\left(e^{-i k z \cdot \widehat{\theta}_{1}}, \ldots, e^{-i k z \cdot \widehat{\theta}_{n}}\right)^{\top}
$$

Then the Tikhonov regularization of (13) leads to solving

$$
\min _{\mathbf{g}_{z} \in \mathrm{C}^{n}}\left\|\Lambda V^{*} \mathbf{g}_{z}-\rho_{z}\right\|_{\ell^{2}}^{2}+\alpha\left\|\mathbf{g}_{z}\right\|_{\ell^{2}}^{2}
$$

where $\alpha>0$ is the Tikhonov regularization parameter. Defining $\mathbf{u}_{z}=V^{*} \mathbf{g}_{z}$, we see that the solution to the problem is

$$
u_{z, \ell}=\frac{\sigma_{\ell}}{\sigma_{\ell}^{2}+\alpha} \rho_{z, \ell}, \quad 1 \leq \ell \leq n
$$

and hence

$$
\left\|\mathbf{g}_{z}\right\|_{\ell^{2}}=\left\|\mathbf{u}_{z}\right\|_{\ell^{2}}=\left(\sum_{\ell=1}^{n} \frac{\sigma_{\ell}^{2}}{\left(\alpha+\sigma_{\ell}^{2}\right)^{2}}\left|\rho_{z, \ell}\right|^{2}\right)^{1 / 2}
$$

The regularization parameter $\alpha$ depends on both $z$ and the error in the data $\left\{u_{\infty}^{a}\right\}$ [5]. As mentioned previously, we use the Morozov discrepancy principle 
to determine $\alpha$. Suppose we know an estimate for the error in the far field operator so that

$$
\left\|F-F_{h}^{a}\right\| \leq \delta
$$

for some $\delta>0$ (using the operator norm induced by $L^{2}\left(\mathrm{~S}^{2}\right)$ ). Then the Morozov procedure picks $\alpha=\alpha(z)$ to be the zero of

$$
m_{z}(s)=\sum_{j=1}^{n} \frac{\delta^{2} \sigma_{j}^{2}-s^{2}}{\left(s+\sigma_{j}^{2}\right)^{2}}\left|\rho_{z, j}\right|^{2}, \quad s>0 .
$$

For the second variant, (15) yields the linear system

$$
V \Lambda^{1 / 2} V^{*} \mathbf{g}_{z}=\mathbf{r}_{z}
$$

If we define $\mu_{z}=V^{*} \mathbf{r}_{z}$ and $\mathbf{v}_{z}=V^{*} \mathbf{g}_{z}$, the solution of this equation is

$$
v_{z, \ell}=\mu_{z, \ell} \sigma_{\ell}^{-1 / 2}, \quad 1 \leq \ell \leq n
$$

and the norm of the solution becomes

$$
\left\|\mathbf{g}_{z}\right\|_{\ell^{2}}=\left(\sum_{\ell=1}^{n} \frac{\left|\mu_{z, \ell}\right|^{2}}{\sigma_{l}}\right)^{1 / 2} .
$$

For the shape reconstruction we proceed as follows. For some $\varepsilon>0$ we determine the boundary of $\Omega_{n}^{\varepsilon}$. Since the evaluation for each point requires 
the solution of a linear system, we use an adaptive strategy to minimize the costs. We will comment on the choice of the parameter $\varepsilon$ soon.

We choose an initial coarse grid such this grid completely contains the scatterer (see also [2]). In our case this is an $8 \times 8 \times 8$ grid of cubes, but in general the size of the initial grid needs to be chosen so that it is likely that one of the initial vertices will be interior to the objects to be detected (some a priori knowledge of the size of the object to be detected is assumed). We evaluate $\left\|\mathbf{g}_{z}\right\|_{l^{2}}$ for $z$ at the vertices of all the cubes. Then we set $M$ to be the minimum of all these values. The cubes are then classified as follows. If $Q$ is a cube in the mesh then:

- $Q$ is an interior cube if $\varepsilon\left\|\mathbf{g}_{z}\right\|_{l^{2}}<M$ at all the vertices of $Q$.

- $Q$ is an exterior cube if $\varepsilon\left\|\mathbf{g}_{z}\right\|_{l^{2}}>M$ at all the vertices of $Q$.

- $Q$ is a boundary cube if it is not interior or exterior.

At the next stage of the algorithm, each boundary cube is further subdivided into 8 (or $2 \times 2 \times 2$ ) sub-cubes, and the sub-cubes are again classified. In our examples, a third subdivision is also used. Effectively, we have the accuracy of a uniform $32 \times 32 \times 32$ grid but with much less work. A typical refinement path (for the star) is shown in Figure 3. In the final step of the surface reconstruction algorithm we proceed as follows: For every boundary cell we use linear interpolation along the edges to locate points where $\varepsilon\left\|\mathbf{g}_{z}\right\|_{\ell^{2}}=$ $M$. Then we draw a polyhedral using these points as vertices. This results 
in a more accurate approximation of the boundary than a simple "box" approximation using boundary cubes. The use of the adaptive threshold algorithm alleviates the three problems mentioned previously in that the algorithm efficiently predicts a surface regardless of topology or position. However the method depends critically on the choice of the cut-off parameter $\varepsilon$. Numerical experiments reveal that $\varepsilon$ needs to be chosen depending on the method (variant (I) or (II)), wave numbers, and size of the target scatterer. Thus we propose to "calibrate" each method by choosing $\varepsilon$ using a synthetic experiment with a known scatterer. In our experiments, we use a unit ball as the calibration scatterer. Using the wave number for the inverse problem, we can predict the far field pattern for the calibration scatterer, then compute $\left\|\mathbf{g}_{z}\right\|_{\ell^{2}}$ in the desired region of space for the inverse problem (the ball is useful since the far field is easy to compute via series). We then choose $\varepsilon$ such that the surface where

$$
\varepsilon\left\|\mathbf{g}_{z}\right\|_{\ell^{2}}=\min \left\|\mathbf{g}_{w}\right\|_{\ell^{2}}
$$

matches the boundary of the calibration scatterer.

Comparisons of $1 /\left\|\mathbf{g}_{z}\right\|$ for the various methods are shown in the first row of Figure 4. It is clear that $\varepsilon$ for the variant (I) must be chosen larger than $\varepsilon$ for the variant (II), and that the choice depends on $k$. We also see that for the sound hard case and for large wavenumber $k$ the sampling yields no sharp jumps near the boundary. To overcome this problem we replace the right hand side $r_{z}$ with the farfield pattern of higher moments, e.g., with the 

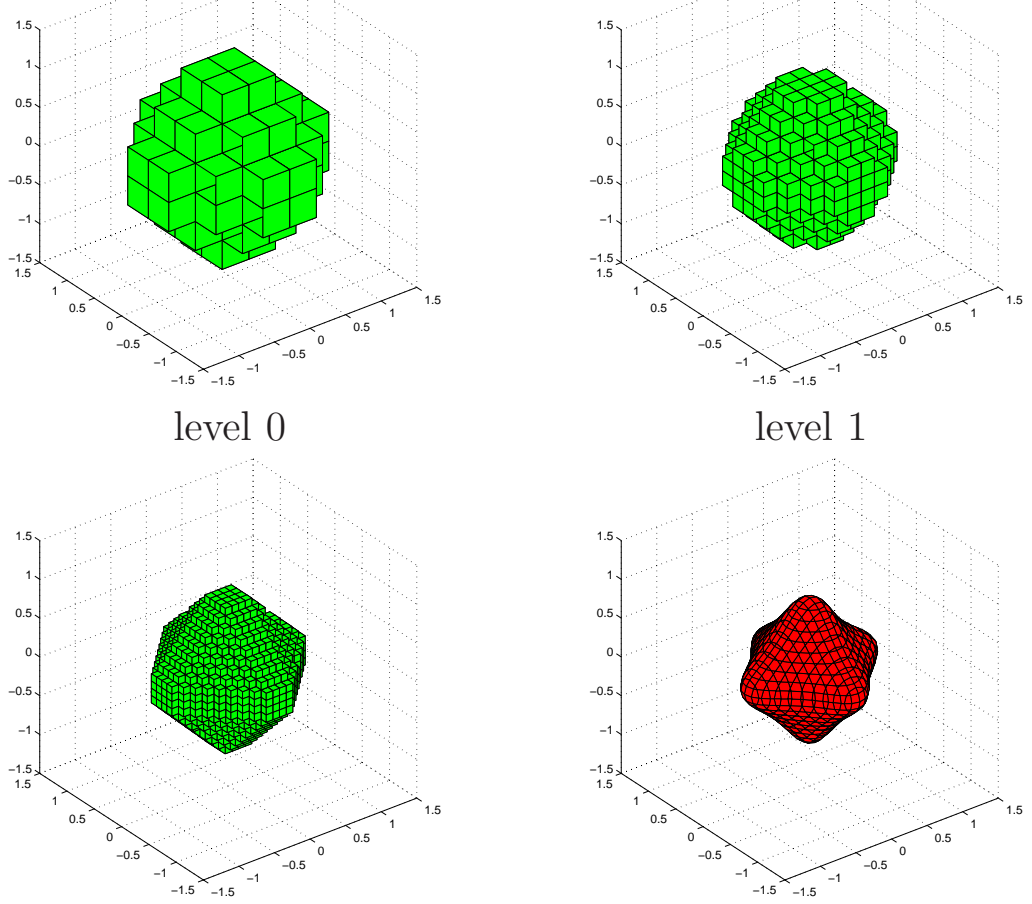

level 2

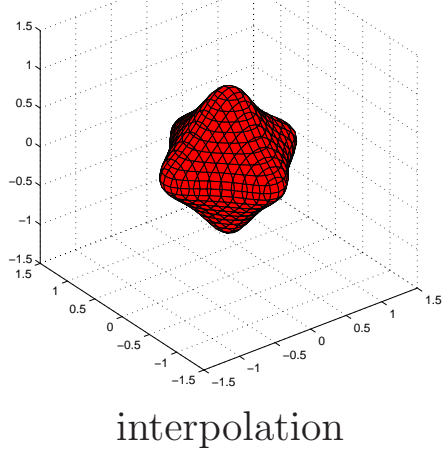

FiguRE 3: Here we show three steps of the adaptive reconstruction algorithm applied to star shaped scatterer 
farfield pattern of an acoustic dipole

$$
\Psi_{z}(x)=d \cdot \nabla \frac{e^{i k|x-z|}}{|x-z|}, \quad d \in \mathrm{S}^{2}
$$

which gives the farfield pattern

$$
r_{z}(\widehat{x})=i k \widehat{x} \cdot d e^{-i k z \cdot \widehat{x}} .
$$

The second row in Figure 4 shows the result for this modified right hand side.

\section{$5 \quad$ Numerical Results}

In this section we shall further develop the LSM algorithms, and give some results of numerical experiments that illustrate properties of the LSM.

The numerical results all use synthetic data computed using a Galerkin boundary element method based on piecewise linear finite elements due to Giebermann (see [8] for details of the implementation of the method and an analysis of its convergence characteristics).

From the Galerkin scheme, we obtain an approximation $u_{\infty}^{B E M}$ of $u_{\infty}$ at the measurement points $\widehat{\theta}_{j}, j=1, \ldots, n$. Having computed this finite element far field pattern, we add noise to the data by defining

$$
u_{\infty}^{a}\left(\widehat{\theta}_{j}, \widehat{\theta}_{\ell}\right)=u_{\infty}^{B E M}\left(\widehat{\theta}_{j}, \widehat{\theta}_{\ell}\right)+\theta \chi_{j, \ell}, 1 \leq j, \ell \leq n
$$


sound soft

$$
k=2
$$
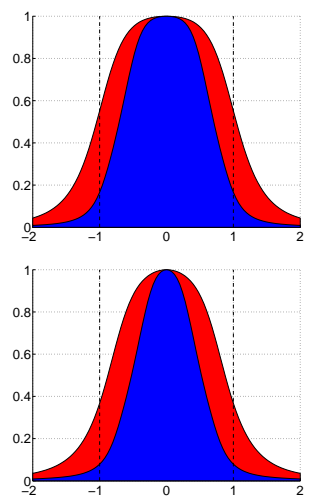

sound hard

$$
k=2
$$
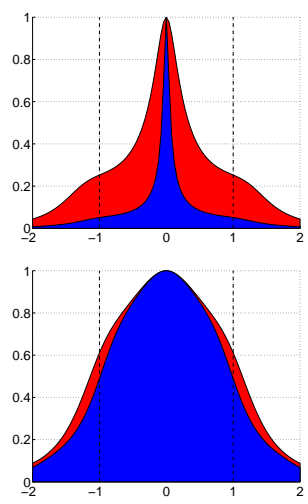

sound soft

$$
k=4
$$
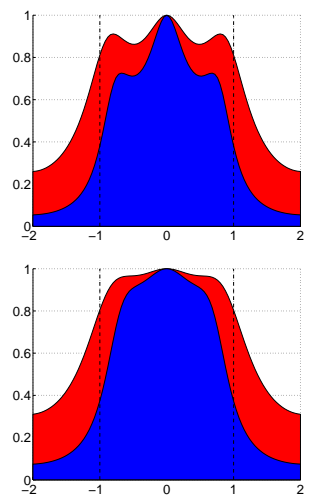

sound hard

$$
k=4
$$
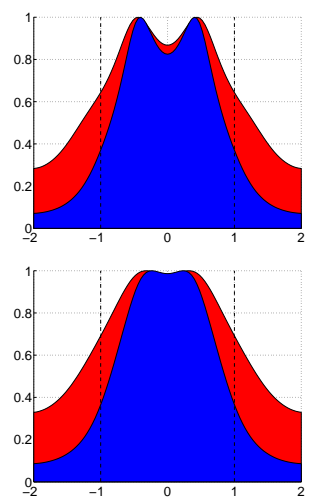

Figure 4: Here we show plots of $1 /\|\mathbf{g}\|_{l^{2}}$ as a function of $x \in[-2,2]$ for $y=z=0$ using the unit sphere calibration scatterer. The values have been normalized so that the maximum is unity. The best choice of cut-off parameter is easy to find since we know the geometry. The first row displays the results for the farfield pattern of a point source as right hand side, the second row shows the results for the farfield pattern of a dipole. Legend: variant (I), $\square$ variant (II) 
where $\chi_{\ell, m}$ is drawn from a sequence of uniformly distributed random numbers in $(-1,1)$. The error parameter $\theta$ is chosen so that if $A^{B E M}$ is the matrix for the data $u_{\infty}^{B E M}$ and if $A$ is the matrix using $u_{\infty}^{a}$, then

$$
\left\|A-A^{B E M}\right\|=0.05
$$

using the spectral norm. Thus we introduce $5 \%$ noise on the finite element solution and choose $\delta=0.05$. This is likely to underestimate the true error in the data.

Figure 6 shows the results for the reconstruction of various objects. The first column displays the original shape, the second one displays the reconstruction due to variant (I) and the last column shows the reconstruction by using variant (II). All reconstructions are for sound soft obstacles.

In the first row we present the reconstruction of a star like region where the measurement was taken for 42 directions and 5\% noise. The second row displays the results for a dolphin and the third row displays that several objects can be reconstructed. Finally, the last row displays the reconstruction of an object which has a rather complicated topological structure.

Finally, Figure 5 displays the results for the reconstruction of penetrable objects for $k=2$. Here, the refractive index is $n(x)=1-I\left(\left|x-x_{1}\right|\right)-\frac{1}{2} I(\mid x-$ $\left.x_{2} \mid\right)$ with the inclusion $I(r)=1 / 2(1+\cos (2 \pi r))$ for $r \leq 1 / 2$ and $I(r)=0$ for $r>1 / 2$. For the reconstruction we use the scattered field $u^{s}$ instead of $u_{\infty}$ as data. In contrast to our previous experiments, the measurements are take for the scattering of point sources located above the objects. Although we 
Original

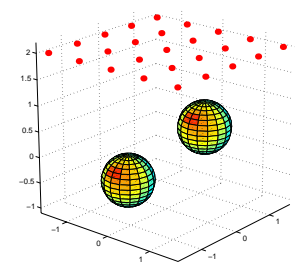

variant (I)

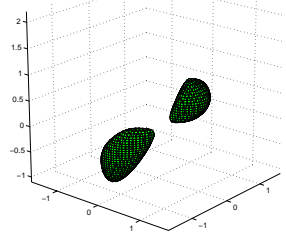

variant (II)

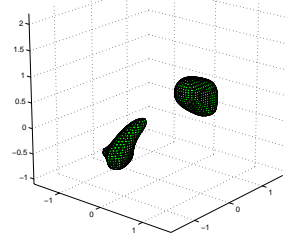

FiguRE 5: This figure shows the reconstruction of two penetrable objects without added noise. Here, the measurements are taken from the scattering of $5 \times 5$ point sources located above the objects.

have a limited apparature, the linear sampling methods gives good results even in the lower parts of the objects.

In order to apply the linear sampling method the nearfield measurements, we replace the farfield operator $F$ by an operator $N$ which has the nearfield data as a kernel. Then we can proceed in the same manner as for the farfield operator.

\section{Conclusion}

We have proposed an efficient scheme for implementing the linear sampling methods for solving the inverse problem. We have demonstrated that discon- 
Original shape
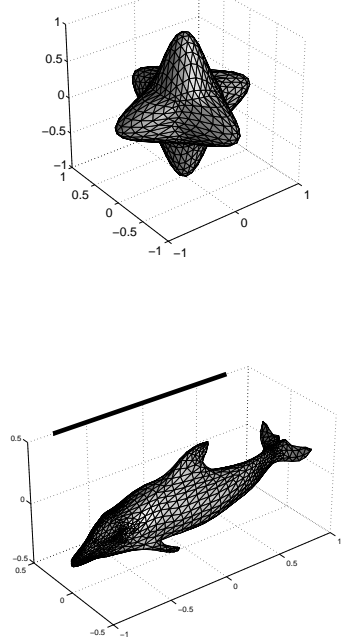

variant (I)

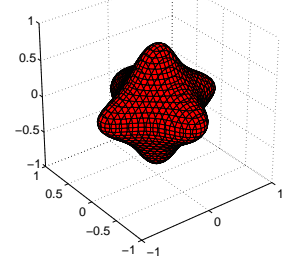

$$
k=2, n=42,5 \% \text { noise }
$$

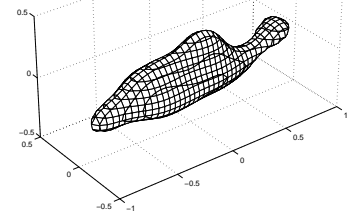

\section{variant (II)}
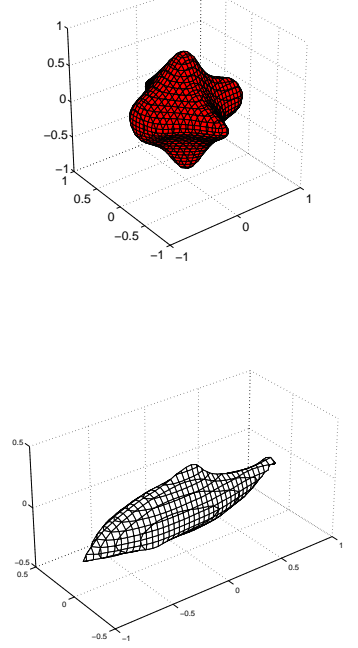

$$
k=4, n=162,0 \% \text { noise }
$$

Figure 6: Here, and continued on the next page, we show the results for the linear sampling method for the reconstruction of various sound soft obstacles. The first column displays the original shape of the scatterer, whereas the second and third column shows the reconstruction due to the variant (I) and (II) respectively. 
Original shape
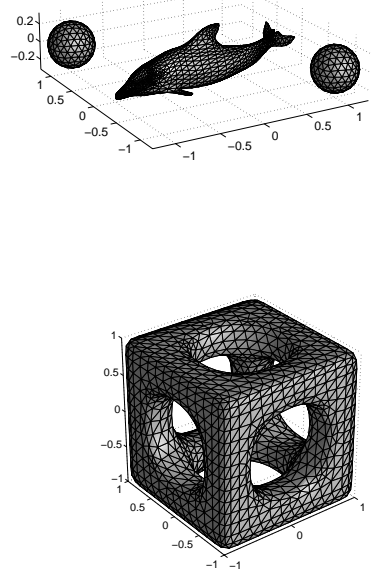

variant (I)

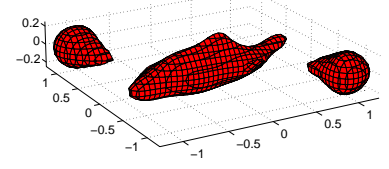

$k=4, n=162,5 \%$ noise

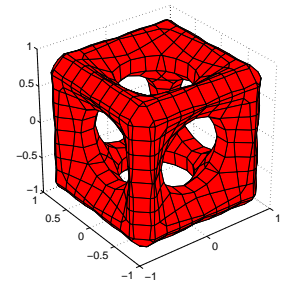

$k=4, n=162,0 \%$ noise variant (II)
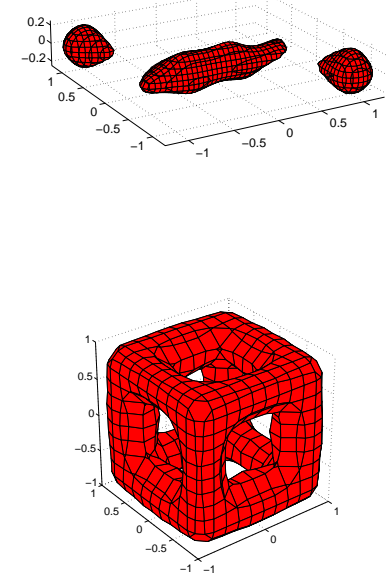

Figure 6: continued. 
nected scatterers as well as complicated topologies can be handled without difficulty. Furthermore, we have shown that the method can also solve the inverse scattering problem for penetrable objects.

Acknowledgements: This research was sponsored by the Air Force Office of Scientific Research, Air Force Materials Command, USAF, grant F4962098-1-0019, and supported by SFB 256, University of Bonn.

\section{References}

[1] T. S. Angell, X. Jiang, and R. E. Kleinman. A distributed source method for inverse acoustic scattering. Inverse Problems, 13:531-545, 1997.

[2] D. Colton and A. Kirsch. A simple method for solving inverse scattering problems in the resonance region. Inverse Problems, 12:383-393, 1996. C436 C436, C437, C440, C448

[3] D. Colton and R. Kress. Inverse Acoustic and Electromagnetic Scattering Theory, no. 93. In Applied Mathematical Sciences. Springer-Verlag, New York, second ed., 1998. C436, C437 
[4] _ The numerical solution of the three dimensional inverse scattering problem for time-harmonic acoustic waves. SIAM J. Sci. Stat. Comp., 8:278-291, 1987. C436

[5] D. Colton, M. Piana, and R. Potthast. A simple method using Morozov's discrepancy principle for solving inverse scattering problems. Inverse Problems, 13:1477-1493, 1997. C436, C437, C440, C442, C446

[6] D. Colton and P. Monk. A linear sampling method for the detection of leukemia using microwaves. SIAM J. on Applied Math, 58:926-941, 1998. C440

[7] D. Colton, K. Giebermann, and P. Monk. The regularized sampling method. SIAM J. Sci. Stat. Comp., to appear. C437, C440

[8] K. Giebermann. Fast Summation Methods for the Numerical Solution of Integral Equations for Scattering Problems in $\mathrm{R}^{3}$, $\mathrm{PhD}$ thesis, University of Karlsruhe, 1997. C451

[9] M. Haas, W. Rieger, W. Rucker, and G. Lehner. Inverse $3 d$ acoustic and electromagnetic obstacle scattering by iterative adaption. In G. Chavent and P. Sabatier, editors, Inverse Problems in Wave Propagation and Diffractioni, pp. 204-215. Berlin, 1997, Springer. C436

[10] A. Kirsch. Factorization of the far field operator for the inhomogeneous medium case and an application in inverse scattering theory. Inverse Problems, to appear. C443 
[11] A. Kirsch. Characterization of the shape of the scattering obstacle by the spectral data of the far field operator. Inverse Problems, 14:1489-1512, 1998. C442, C443

[12] R. Kress and A. Zinn. On the numerical solution of the three dimensional inverse obstacle scattering problem. J. Comp. Appl. Math., 42:49-61, 1992. C436

[13] P. Maponi, L. Misici, and F. Zirilli. An inverse problem for the three dimensional vector Helmholtz equation for a perfectly conducting obstacle. Computers Math. Application, 22:137-146, 1991. C436

[14] P. Maponi, M. Recchioni, and F. Zirilli. The use of optimization in the reconstruction of obstacles from acoustic or electromagnetic scattering data. In L. Biegler, et. al., editors, Large Scale Optimization with Applications, pp. 81-100. IMA Volumes in Mathematics and its Applications, New York, 1997, Springer. C436

[15] L. Misici and F. Zirilli. Three dimensional inverse obstacle scattering for time harmonic acoustic waves: a numerical method. SIAM J. Sci. Stat. Comp., 15:1174-1189, 1994. C436

[16] A. Norris. A direct inverse scattering method for imaging obstacles with unknown surface conditions. IMA Jour. Applied Math., 61:267-290, 1998. C436, C437 
[17] R. Potthast. A point-source method for inverse acoustic and electromagnetic obstacle scattering problems. IMA Jour. Applied. Math., to appear. C436

[18] _ _. A fast new method to solve inverse scattering problems. Inverse Problems, 12:731-742, 1996. C436

[19] A. N. Tikhonov, A. V. Goncharsky, V. V. Stepanov, and A. G. Yagola. Numerical Methods for the Solution of Ill-Posed Problems. Kluwer, Dordrecht, 1995. 